\title{
Low-energy electron-impact excitation of the hydrogen molecule*
}

\author{
T. N. Rescigno, ${ }^{\dagger}$ C. W. McCurdy, Jr., ${ }^{\ddagger}$ and V. McKoy \\ Arthur Amos Noyes Laboratory of Chemical Physics, §.California Institute of Technology, Pasadena, California 91125 \\ C. F. Bender" \\ Lawrence Livermore Laboratory, University of California, Livermore, California 94550
}

(Received 8 September 1975)

\begin{abstract}
We present cross sections for the excitation of the two lowest $\left(b^{3} \Sigma_{u}^{+}\right.$and $\left.a^{3} \Sigma_{g}^{+}\right)$triplet states of molecular hydrogen by electron impact for incident electron energies $\leq 20 \mathrm{eV}$. The cross sections are calculated using the distorted-wave approximation with the inelastic transition density obtained in the random-phase approximation. An efficient expansion technique using Gaussian basis functions allows us to avoid numerical integrations and to treat accurately the noncentral nature of the scattering process with full allowance for electron exchange. The sum of the two triplet cross sections is found to be in good agreement with the experimental cross section for dissociation of $\mathrm{H}_{2}$ into $2 \mathrm{H}$.
\end{abstract}

\section{INTRODUCTION}

Current efforts in the identification and development of new gas-laser systems have sparked a renewed interest in gas dynamic processes and, in particular, have created a need for accurate lowenergy electron-molecule scattering cross sections. The precise evaluation of cross sections for processes involving the collision of slow electrons with molecules has been greatly hindered in the past because of the severe difficulties introduced into the calculation of molecular continuum wave functions by the nonspherical nature of the molecular force field. In most cases, numerical complications have restricted the computations to a rather low level of approximation; indeed, for cases involving molecular electronic excitation, the calculations almost invariably fall under the category of Born-type calculations in the case of spin-allowed transitions, or in the case of singlettriplet transitions, Born-Oppenheimer or Ochkurtype calculations. ${ }^{1}$ Experience gained in the calculation of electron-atom cross sections has shown that the validity of such approximate schemes at low electron impact energies $(<50 \mathrm{eV})$ is highly questionable. ${ }^{2,3}$

In a previous Letter, ${ }^{4}$ we reported differential cross sections for the dissociative $b^{3} \Sigma_{u}^{+}$state of $\mathrm{H}_{2}$ at $15 \mathrm{eV}$ incident electron energy. The results were in good agreement with experimental data. The computations were carried out using the socalled "first-order many-body formula" ${ }^{5}$ wich, as the authors have shown, ${ }^{6}$ is simply a form of the distorted-wave approximation in which both the incident and scattered electron move in the field of the initial electronic state of the target. The distorted waves were obtained by an efficient expansion technique which involves solving the LippmannSchwinger equation for the molecular continuum orbitals in a discrete set of Gaussian basis functions. ${ }^{7,8}$ This approach allowed us to treat the noncentral nature of the scattering process by explicitly including the $\Sigma_{g}^{+}, \Sigma_{u}^{+}, \Pi_{g}$, and $\Pi_{u}$ contributions to the transition amplitude without resorting to $\mathrm{a}^{\text {. }}$ one-center expansion. The fact that no numerical integrations were performed also enabled us to treat the exchange forces properly in computing the distorted waves.

These computations have now been extended to other energies between 13 and $20 \mathrm{eV}$. We have also considered the excitation of the second $\left(a^{3} \Sigma_{g}^{+}\right)$triplet state of $\mathrm{H}_{2}$ over the same energy range. As will be shown, these two cross sections can be combined to give a total cross section for the dissociation of $\mathrm{H}_{2}$ that is in good agreement with the experimental cross section over this range of electron impact energies. The general theory and approximation schemes employed in these calculations will also be considered in some detail.

\section{THEORY}

A. Separation of electronic, vibrational and rotational motion

Within the framework of the Born-Oppenheimer approximation, we denote the electronic, vibrational, and rotational quantum numbers of the initial (final) state of the electron $-\mathrm{H}_{2}$ system by $n, \nu$, and $l$ and $m\left(n^{\prime}, \nu^{\prime}, l^{\prime}, m^{\prime}\right)$ and write the wave function for this state as

$$
\Psi_{i(f)}^{\text {total }}=\Psi_{n\left(n^{\prime}\right)}^{+(-)}(\{\overrightarrow{\mathrm{x}}\}, \overrightarrow{\mathrm{R}}) \zeta_{\nu\left(v^{\prime}\right)}^{n\left(n^{\prime}\right)}(R) Y_{l\left(l^{\prime}\right)}^{m\left(m^{\prime}\right)}(\theta, \varphi),
$$

where $\Psi_{n}^{+}$, the electronic wave function, depends explicitly on the electronic space and spin coordinates $\{\vec{x} \equiv \vec{r}, \vec{s}\}$ in a body-fixed coordinate system and on the internuclear separation $R$. There is also an implicit dependence on the orientation of the molecule with respect to a laboratory-fixed frame, which comes about through the imposition of as- 
ymptotic, scattering boundary conditions. The vibrational wave function $\zeta_{\nu}^{n}$ is assumed to be independent of the rotational quantum numbers $l$ and $m$ and $Y_{l}^{m}(\theta, \varphi)$ is a spherical harmonic, with polar angles referring to the orientation of the molecular axis with respect to the laboratory frame.

If we label the incident electron as particle 1 , the cross section for the excitation of a particular electronic/vibrational/rotational state is given (in atomic units) by

$$
\begin{aligned}
\sigma\left(\overrightarrow{\mathrm{k}}_{f}-\overrightarrow{\mathrm{k}}_{i}\right)=\frac{1}{4 \pi^{2}} \frac{k_{f}}{k_{i}} \mid & \int e^{-i \overrightarrow{\mathrm{k}}_{f} \vec{o}_{I}} \chi^{*}\left(\overrightarrow{\mathrm{s}}_{1}\right) \Phi_{n^{\prime}}\left(\overrightarrow{\mathrm{x}}_{2}, \overrightarrow{\mathrm{x}}_{3} ; R\right) \zeta \nu_{\nu^{\prime}}^{n^{\prime}}(R) Y_{l^{\prime}}^{m^{\prime}}(\theta, \varphi)\left(-\frac{1}{r_{1 A}}-\frac{1}{r_{1 B}}+\frac{1}{r_{12}}+\frac{1}{r_{13}}\right) \Psi_{n}^{+}\left(\overrightarrow{\mathrm{x}}_{1}, \overrightarrow{\mathrm{x}}_{2}, \overrightarrow{\mathrm{x}}_{3} ; \overrightarrow{\mathrm{R}}\right) \\
& \times\left.\zeta_{\nu}^{n}(R) Y_{l}^{m}(\theta, \varphi) d \overrightarrow{\mathrm{x}}_{1} d \overrightarrow{\mathrm{x}}_{2} d \overrightarrow{\mathrm{x}}_{3} d \overrightarrow{\mathrm{R}}\right|^{2},
\end{aligned}
$$

where $\chi\left(S_{1}\right)$ is the spin wave function of the scattered electron, $\Phi_{n^{\prime}}$ is the normalized final-state electronic wave function of $\mathrm{H}_{2}$, and $A$ and $B$ denote the two hydrogen nuclei. $\overrightarrow{\mathrm{k}}_{i}$ and $\overrightarrow{\mathrm{k}}_{f}$, the wave vectors for the incident and scattered electrons, are determined by the relation

$$
k_{f}^{2}=k_{i}^{2}-2\left(E_{f}-E_{i}\right)
$$

where $E_{f}\left(E_{i}\right)$ is the final (initial) energy of the molecule. In defining the cross section we have used the post-interaction representation ${ }^{9}$ in which the noninteracting form of $\Psi_{n^{\prime}}^{-}$appears, and $\Psi_{n}^{+}(\{\overrightarrow{\mathrm{x}}\}, R)$ is the fully antisymmetrized outgoing electronic wave function for the $e^{-}-\mathrm{H}_{2}$ system.

We can first treat the contribution to the cross section arising from the rotational motion of the molecule. Since rotational levels are typically not resolved in current experiments, it is customary to treat them as being effectively degenerate and to sum the cross section of Eq. (2) over all final rotational levels $l^{\prime}$ and $m^{\prime}$ and average over all ini tial states $l$ and $m$. If we also neglect the slight dependence of the molecular energy upon $l$ and $l^{\prime}$, it then follows, from the completeness properties of the spherical harmonics, that the rotationally averaged cross section can be written as ${ }^{10}$

$$
\begin{aligned}
\sigma^{\text {rot. av. }}\left(k_{f}-k_{i}\right)=\frac{1}{4 \pi^{2}} \frac{k_{f}}{k_{i}} \int \mid & \int e^{-i \overrightarrow{\mathrm{k}}_{f} \cdot \overrightarrow{\mathrm{r}}_{i}} \chi^{*}\left(\overrightarrow{\mathrm{S}}_{1}\right) \Phi_{n^{\prime}}^{*}\left(\overrightarrow{\mathrm{x}}_{2}, \overrightarrow{\mathrm{x}}_{3} ; R\right) \zeta_{\nu^{\prime}}^{n^{n^{\prime}}}(R)\left(-\frac{1}{r_{1 A}}-\frac{1}{r_{1 B}}+\frac{1}{r_{12}}+\frac{1}{r_{13}}\right) \Psi_{n}^{+}\left(\overrightarrow{\mathrm{x}}_{1}, \overrightarrow{\mathrm{x}}_{2}, \overrightarrow{\mathrm{x}}_{3} ; \overrightarrow{\mathrm{R}}\right) \\
& \times\left.\zeta_{\nu}^{n}(R) R^{2} d R d \overrightarrow{\mathrm{x}}_{1} d \overrightarrow{\mathrm{x}}_{2} d \overrightarrow{\mathrm{x}}_{3}\right|^{2} \frac{d \Omega}{4 \pi}
\end{aligned}
$$

This result, which is equivalent to an adiabatic treatment of molecular rotation, ${ }^{11}$ simply amounts to averaging the cross section over all possible orientations of the molecular target.

A further approximation may be made by realizing that the electronic wave functions are more slowly varying functions of the internuclear separation $R$ than the vibrational wave functions and hence replacing the former by their values at the equilibrium internuclear separation. This approximation works best when the initial vibrational state is $\nu=0$ (which is the case for hydrogen at room temperature) since the vibrational wave func- tion is then nearly Gaussian and centered about the equilibrium internuclear separation $R_{e}$. The vibrational component of the cross section can then be separated from Eq. (4) and gives a multiplicative factor of

$$
\boldsymbol{q}_{\nu^{\prime} \nu} \equiv\left|\int R \zeta_{\nu^{\prime}}^{n^{\prime} *}(R) R \zeta_{\nu}^{n}(R) d R\right|^{2},
$$

which is the familiar Franck-Condon overlap integral. ${ }^{10}$ The cross section for exciting all the vibrational levels of a particular electronic state can then be obtained by summing over all $\nu^{\prime}$ values to give

$$
\begin{aligned}
\sigma_{\text {total }}^{\text {rot. av. }}\left(\overrightarrow{\mathrm{k}}_{f}-\overrightarrow{\mathrm{k}}_{i}\right)=\sum_{\nu^{\prime}} \frac{1}{4 \pi^{2}} \frac{k_{f}}{k_{i}} q_{\nu \nu^{\prime}} \int \mid \int e^{-i \overrightarrow{\mathrm{k}}_{f^{\prime}} \overrightarrow{\mathrm{r}}_{i}} \chi^{*}\left(\overrightarrow{\mathrm{s}}_{1}\right) \Phi_{n^{\prime}}^{*}\left(\overrightarrow{\mathrm{x}}_{2}, \overrightarrow{\mathrm{x}}_{3} ; R_{e}\right)\left(-\frac{1}{r_{1 A}}-\frac{1}{r_{1 B}}+\frac{1}{r_{12}}+\frac{1}{r_{13}}\right) \\
\times \Psi_{n}^{+}\left(\overrightarrow{\mathrm{x}}_{1}, \overrightarrow{\mathrm{x}}_{2}, \overrightarrow{\mathrm{x}}_{3} ; \overrightarrow{\mathrm{R}}_{e}\right) d \overrightarrow{\mathrm{x}}_{1}, d \overrightarrow{\mathrm{x}}_{2},\left.d \overrightarrow{\mathrm{x}}_{3}\right|^{2} \frac{d \Omega}{4 \pi}
\end{aligned}
$$

A final approximation consists of ignoring the dependence of the electronic portion of the scattering amplitude on the vibrational quantum number; that is, for each value of the incident electron energy under consideration, a single electronic tran- sition amplitude was calculated, with a final wave number corresponding to a vertical transition from the $\nu=0$ ground state into the vibrational level of the upper electronic state with the largest FranckCondon overlap. Using the electronic potential- 
energy curves of Kolos and Wolniewicz, ${ }^{12,13}$ this corresponds to a vertical transition energy of 10.14 $\mathrm{eV}$ for the $b^{3} \Sigma_{u}^{+}$state and $12.12 \mathrm{eV}$ for the $a^{3} \Sigma_{g}^{+}$ state. Relaxing this approximation would have required computing a different electronic transition matrix for every possible final vibrational statea procedure that would have involved a consider able amount of computer time. The final form of the differential, rotationally averaged, total vibrational cross section used in this work is

$$
\sigma_{\text {total }}^{\text {rot. av. }}=\frac{1}{4 \pi^{2}} \int\left|T_{\mathrm{el}}\left(\overrightarrow{\mathrm{k}}_{f}-\overrightarrow{\mathrm{k}}_{i}\right)\right|^{2} \frac{d \Omega}{4 \pi} \sum_{\nu^{\prime}} \frac{k_{f}}{k_{i}} q_{\nu \nu^{\prime}},
$$

where

$$
\begin{aligned}
T_{\mathrm{el}}\left(\overrightarrow{\mathrm{k}}_{f}-\overrightarrow{\mathrm{k}}_{i}\right)= & \int e^{-i \overrightarrow{\mathrm{k}}_{f}\left(R_{e}\right) \cdot \overrightarrow{\mathrm{r}}_{i}} \chi^{*}\left(s_{1}\right) \Phi_{n^{\prime}}^{*}\left(\overrightarrow{\mathrm{x}}_{2}, \overrightarrow{\mathrm{x}}_{3} ; R_{e}\right) \\
& \times\left(-\frac{1}{r_{1 A}}-\frac{1}{r_{1 B}}+\frac{1}{r_{12}}+\frac{1}{r_{13}}\right) \\
& \times \Psi_{n}^{+}\left(\overrightarrow{\mathrm{x}}_{1}, \overrightarrow{\mathrm{x}}_{2}, \overrightarrow{\mathrm{x}}_{3} ; \overrightarrow{\mathrm{R}}_{e}\right) d \overrightarrow{\mathrm{x}}_{1} d \overrightarrow{\mathrm{x}}_{2} d \overrightarrow{\mathrm{x}}_{3} .
\end{aligned}
$$

In the case of the dissociative $b^{3} \Sigma_{u}^{+}$state, the sum over $\nu^{\prime}$ in Eq. (7) is replaced by an integral.

B. Distorted-wave-random-phase approximation (DW-RPA) for the electronic transition matrix

For the computations reported in this paper, the electronic transition matrix $T_{\mathrm{el}}$ was calculated using the first-order many-body formula. This approximation was first introduced into electron scattering by Taylor and his co-worker $\mathrm{s}^{5}$ and ap- plied to $e^{-}$-He scattering. ${ }^{3}$ As the present authors have shown, ${ }^{6}$ the first-order many-body formula is simply a form of the distorted-wave (DW) approximation in which both distorted waves are computed in the field of the initial state and the electronic target states are treated in the random-phase approximation (RPA). Other DW calculations have appeared in the literature recently, on ${ }^{14} e^{-}-\mathrm{He}$ and $e^{-}-\mathrm{H},{ }^{15}$ and in both studies it was shown that better results were obtained when the initial- and finalstate orbitals were both computed in the field of the ground state-in agreement with DW-RPA prescription.

The DW-RPA ${ }^{6}$ can be easily obtained for the general case of scattering from an $n$-electron closed-shell atom or molecule by using the twopotential formula. ${ }^{16}$ To the interaction potential $V$ between an incident electron and an $n$-electron atom or molecule, we add and subtract the operators $J-K$, the Hartree-Fock Coulomb and exchange operators for the target in its ground state. We then partition $V$ into two terms, $V=V_{I}+V_{\mathrm{II}}$, where

$$
V_{\mathrm{I}}=\sum_{\text {nuclei }}-\frac{Z_{N}}{\left|r-R_{N}\right|}+J-K
$$

and

$$
V_{\mathrm{II}}=\sum_{i=1}^{n} \frac{1}{\left|r-r_{i}\right|}-J+K .
$$

We may then write the electronic transition matrix element for inelastic scattering as

$$
T_{\mathrm{el} 1}\left(\overrightarrow{\mathrm{k}}_{f}-\overrightarrow{\mathrm{k}}_{i}\right)=\left(\Phi_{n^{\prime}}\left(x_{1}, \ldots, x_{n}\right) e^{\overrightarrow{\mathrm{k}}_{f_{f^{\mathrm{T}}}}} \chi(s)\left|V_{\mathrm{I}}\right| \Psi_{\mathrm{I} k_{i}}^{+}\left(x, x_{1}, \ldots, x_{n}\right)\right)+\left(\Psi_{\mathrm{I}_{f}}^{-}\left(x, x_{1}, \ldots, x_{n}\right)\left|V_{\mathrm{II}}\right| \Psi^{+}\left(x, x_{1}, \ldots, x_{n}\right)\right)
$$

where $\Psi^{+}$is the exact, antisymmetric electronic wave function for the full interacting system with outgoing-wave boundary conditions, and $\Psi_{1 k_{i}}^{+}$and $\Psi_{\mathrm{I} k_{f}}^{-}$are eigenfunctions of the operator $-\frac{1}{2} \nabla_{r}^{2}+\boldsymbol{H}_{\text {target }}$ $+V_{\mathrm{l}}$. Thus $\Psi_{1 k_{i}}^{+}$and $\Psi_{I k_{f}}^{+}$are products of target eigenstates and scattering solutions of the static exchange Hamiltonian $-\frac{1}{2} \nabla_{r}^{2}+V_{\mathrm{I}}$. The distortedwave approximation is made by replacing $\Psi^{+}$by an antisymmetrized product of the target ground-state wave function and a Hartree-Fock continuum spin orbital. The choice of the ground state $J-K$ operators in the definition of $V_{\mathrm{I}}$ leads naturally to a distorted-wave approximation in which the distorting potential is the static-exchange potential of the ground state. However this is a somewhat artificial choice whose justification lies mainly in the better agreement obtained for ${ }^{15} e^{-}-\mathrm{H}$ and ${ }^{14} e^{-}-\mathrm{He}$ cross sections using the field of the ground state than were obtained using the field of the excited state. In the theory of Taylor and co-worker $\mathrm{s}^{3,5}$ the replacement of certain operators in the BetheSalpeter equation by their Hartree-Fock values leads to the same final result. In fact this is a result to be expected in first order from a many-body theory approach to scattering which starts from a set of hole and particle (bound and continuum) orbitals which are eigenfunctions of the ground-state Hartree-Fock Hamiltonian. ${ }^{6}$

The distorted-wave approximation to the transition element may be written as a matrix element of a simple operator between the ground and excited states of the target. This expression is then evaluated in the RPA (details are given in Ref. 6) to yield

$$
T_{\mathrm{el}}\left(\overrightarrow{\mathrm{k}}_{f}-\overrightarrow{\mathrm{k}}_{i}\right)=\sum_{m \alpha} Y_{m \alpha}^{*(f)}\left(m k_{f}^{-}|| \alpha k_{i}^{+}\right)_{a}+Z_{m \alpha}^{*^{(f)}}\left(\alpha k_{f}^{-}|| m k_{i}^{+}\right)_{a},
$$

where $Y_{m \alpha}^{(f)}$ and $Z_{m \alpha}^{(f)}$ are amplitudes satisfying the usual RPA equations, $m$ and $\alpha$ refer to Hartree- 
Fock unoccupied and occupied spin orbitals, respectively, $k_{i(f)}^{+(-)}$is a Hartree-Fock continuum spin orbital at the incident (exiting) wave number with outgoing - (incoming-) wave boundary conditions, and the antisymmetrized matrix elements are defined by

$$
(i j|| k l)_{a}=(i j|| k l)-(i j|| l k),
$$

where

$(i j|| k l)=\int \phi_{i}^{*}\left(\overrightarrow{\mathrm{x}}_{1}\right) \phi_{j}^{*}\left(\overrightarrow{\mathrm{x}}_{2}\right) \frac{1}{\left|\overrightarrow{\mathrm{r}}_{1}-\overrightarrow{\mathrm{r}}_{2}\right|} \phi_{k}\left(x_{1}\right) \phi_{l}\left(x_{2}\right) d \overrightarrow{\mathrm{x}}_{1} d \overrightarrow{\mathrm{x}}_{2}$.

For the calculations presented here we require an expression for the cross section in the special case of excitation from a closed-shell singlet ground state to a triplet state. By using the spinadapted excitation operators of Shibuya and McKoy, ${ }^{17}$ and following the derivation of Ref. 6, specializations of Eq. (11) for exciting each of the degenerate components of the excited state are obtained. The cross section is found by summing over components of the final state of the target and over the spin projection of the exiting electron. We assume an unpolarized incident beam and thus average the cross section over spin projections of the incident electron. The resulting expression for the cross section is

$$
\begin{aligned}
& \sigma^{\text {triplet }}\left(\overrightarrow{\mathrm{k}}_{f}-\overrightarrow{\mathrm{k}}_{i}\right) \\
& \quad=\frac{3}{2} \frac{1}{(2 \pi)^{2}} \frac{1}{4 \pi} \int d \Omega\left|T_{\mathrm{el}}^{\text {tripley }}\left(\overrightarrow{\mathrm{k}}_{f}-\overrightarrow{\mathrm{k}}_{i}\right)\right|^{2} \sum_{\nu^{\prime}} \frac{k_{f}}{k_{i}} q_{\nu \nu^{\prime}},
\end{aligned}
$$

where

$$
\begin{aligned}
& T_{\mathrm{el}}^{\mathrm{triplet}}\left(\overrightarrow{\mathrm{k}}_{f}-\overrightarrow{\mathrm{k}}_{i}\right)
\end{aligned}
$$

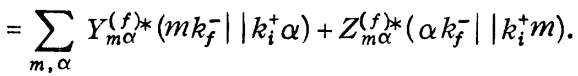

In Eq. (15) $m, \alpha, k_{f}^{-}$and $k_{i}^{+}$are Hartree-Fock space orbitals evaluated at the equilibrium internuclear separation, the spin integrations having been performed in the derivation of Eq. (14).

A similar analysis for exciting a singlet state gives

$$
\begin{aligned}
& \sigma^{\text {singlet }}\left(\overrightarrow{\mathrm{k}}_{f}-\overrightarrow{\mathrm{k}}_{i}\right) \\
& \quad=\frac{1}{2} \frac{1}{(2 \pi)^{2}} \frac{1}{4 \pi} \int d \Omega\left|T_{\mathrm{el}}^{\text {singlet }}\left(\overrightarrow{\mathrm{k}}_{f}-\overrightarrow{\mathrm{k}}_{i}\right)\right|^{2} \sum_{\nu^{\prime}} \frac{k_{f}}{k_{i}} q_{\nu \nu^{\prime}},
\end{aligned}
$$

with

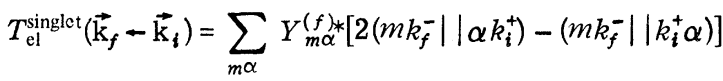

$$
\begin{aligned}
& +Z_{m \alpha}^{(f)}\left[2\left(\alpha k_{f}^{-}|| m k_{i}^{+}\right)-\left(\alpha k_{f}^{-}|| k_{i}^{+} m\right)\right],
\end{aligned}
$$

and where the orbitals are again space orbitals.
C. Evaluation of molecular continuum orbitals

The successful execution of a distorted-wave calculation depends on an efficient technique for computing the continuum orbitals. This is particularly true in molecular applications, where the distorted waves must be computed with a nonspherical Hartree-Fock potential. A partial-wave expansion of the scattering wave function leads to coupled equations whose solutions can, in principle, be found by direct numerical integration. In practice, the relatively slow convergence of single-center expansions ${ }^{18}$ and the difficulties of handling exchange operators render calculations by this method tedious. Other investigators who have chosen this approach have often found it necessary to make severe approximations to the nonlocal portions of the potential to make the calculations tractable. ${ }^{19,20}$

The Hartree-Fock scattering solutions used in this work, $\phi_{k}^{ \pm}(\overrightarrow{\mathrm{r}})$, were computed by direct solution of the Lippmann-Schwinger equation for the wave function. The Lippmann-Schwinger equation can be reduced to a finite matrix equation by expanding the wave function in a set of square-integrable basis functions, which in this case were chosen to be ordinary Gaussian functions, $\varphi_{\alpha}(\overrightarrow{\mathbf{r}})$, centered on the two nuclei ${ }^{7.8}$ :

$$
\phi_{k}^{ \pm}(\overrightarrow{\mathrm{r}})=\sum_{\alpha} \varphi_{\alpha}(\overrightarrow{\mathrm{r}}) c_{\alpha}^{ \pm}(\overrightarrow{\mathrm{k}}) .
$$

The Lippmann-Schwinger equation becomes an algebraic equation for the coefficients $c_{\alpha}^{ \pm}(\overrightarrow{\mathrm{k}})$,

$$
c_{\alpha}^{ \pm}(\overrightarrow{\mathrm{k}})=\langle\alpha \mid \overrightarrow{\mathrm{k}}\rangle+\sum_{\beta, \gamma} G_{\alpha \beta}^{0 \pm} V_{\beta \gamma} c_{\gamma}^{ \pm}(\overrightarrow{\mathrm{k}})
$$

where $V_{B \gamma}$ is a matrix element of the Hartree-Fock potential, $\langle\alpha \mid \overrightarrow{\mathrm{k}}\rangle$ is the Fourier transform of $\varphi_{\alpha}(\overrightarrow{\mathrm{r}})$, and $G_{\alpha \beta}^{0 \pm}$ is a matrix element of the free-particle Green's function. The formulas for the matrix elements in Eq. (19) are given in Refs. 7 and 8. In this approach, nonlocal potential operators pose no more serious problems than in bound-state calculations since only matrix elements of the potential between square-integrable basis functions are required.

The basis-set expansion of $\phi_{k}^{ \pm}(\overrightarrow{\mathrm{r}})$ obtained by the solution of Eq. (10) is only valid over a finite range of $r$, but with a sufficiently large set of basis functions a representation is obtained which can be used to accurately evaluate the matrix elements of Eq. (15). If one chooses basis functions which transform with the ir reducible representations of the point group $D_{\infty h}$, Eq. (19) may be solved separately for each molecular symmetry $\left(\Sigma_{g}^{+}, \Sigma_{u}^{+}, \Pi_{g}\right.$, etc. $)$. This constitutes a major simplification, since it reduces the size of the matrices which must be handled at any point in the calculation. It should be 
noted that the solution of Eq. (19) for $\phi_{\vec{k}}^{ \pm}$is carried out in the body-fixed frame and that the orientation of the molecule with respect to the incident direction $\overrightarrow{\mathrm{k}}$ enters the calculation through the Fourier transforms $\langle\alpha \mid \vec{k}\rangle$. Thus we compute distorted waves for a number of orientations of the molecule and use these to construct the cross section at enough points to perform the average over molecular orientations numerically. The details of this numerical integration in Eq. (14) will be discussed in Sec. III.

We may correct the electronic transition matrix elements in Eq. (15) to a certain extent so as to compensate for the fact that a finite basis representation of $\phi_{\vec{k}}^{ \pm}$is being used. Since the first term in $\phi_{\vec{k}}^{ \pm}[$Eqs. (18) and (19)] is a plane wave, the matrix element (for example) $\left(m \overrightarrow{\mathrm{k}}_{f}^{-}|| \overrightarrow{\mathrm{k}}_{i}^{+} \alpha\right)$ will contain a plane-wave contribution $\left(m \vec{k}_{f}|| \vec{k}_{i} \alpha\right)$. We can substract the basis-set representation of this contribution and replace it by the exact plane-wave matrix element. This substitution also has the effect of including first Born contributions to the scattering amplitude from higher symmetries not included in the basis. ${ }^{8}$ For the basis sets and number of molecular symmetries considered in this work, the plane-wave correction was found to be small in all cases. These plane-wave corrections require the evaluation of free-free matrix elements. Multicenter bound-free and free-free matrix elements can be evaluated in closed form when Gaussian orbitals are used. ${ }^{21,22}$

\section{COMPUTATIONAL}

The first step in the calculation was to compute an accurate Hartree-Fock ground-state wave function and the RPA amplitudes $Y_{m \alpha}$ and $Z_{m \alpha}$ of Eq. (11). For this purpose a $(7 s 5 p)$ set of contracted Gaussian basis functions was used which was formed from the $10 s$ and $5 p$ sets of Huzinaga ${ }^{23}$ (effective Slater exponent of 1.2 ) by contracting the four tightest $s$ functions. The distorted waves $\phi_{\vec{k}}^{ \pm}$of Eq. (18) were then expanded in larger basis sets. Only $\Sigma_{g}^{+}, \Sigma_{u}^{+}, \Pi_{g}$, and $\Pi_{u}$ components of the distorted waves were computed using the LippmannSchwinger equation; higher symmetries were included through the plane-wave corrections, as discussed previously.

For the $\Sigma_{g}^{+}$and $\Sigma_{u}^{+}$components, a $\left(20 s 5 p_{z}\right)$ set of Gaussians on each center was used, with $s$ exponents chosen in a geometric series (most diffuse $\alpha=4 \times 10^{-4}$ ) to extend the Huz inaga ${ }^{23} 10 \mathrm{~s}$ set and $p_{z}$ exponents also chosen in a geometric series (ratio 1.9 , most diffuse $\alpha=0.102$ ). For $\Pi_{g}$ and $\Pi_{u}$ symmetries, a $15 p$ set of primitive Gaussians was formed with exponents ranging from 15.3 to 0.001 . The $\Pi_{x}$ and $\Pi_{y}$ components were solved for sepa- rately but the potential matrix $V_{\alpha \beta}$ in Eq. (19) is the same for both. The adequacy of this basis set was verified by the close agreement obtained at all angles, energies, and orientations between the planewave contributions to the cross section (discussed in Sec. II) and the basis-set representation of these.

Since a smaller basis was used to expand the occupied $\sigma_{g}$ orbital of $\mathbf{H}_{2}$ than was used to expand the distorted waves $\phi_{\mathrm{k}}^{(\neq)}$, considerable economy was realized in the computation of the two-electron integrals which contribute to the potential matrix $V_{\alpha \beta}$ of Eq. (19). The smaller $(7 s 5 p)$ set was also used in solving the RPA equations so that the Hartree-Fock virtual orbitals $m$ in Eq. (15) were also expressed in this basis. Thus only the distorted waves in Eq. (15) are expressed in the larger basis sets and consequently many fewer two-electron integrals are required than would be if the same basis was for both $\varphi_{k}^{( \pm)}$and $m$ and $\alpha$. It is important to realize that the distorted-wave formula [Eq. (15)] can couple the different components of the distorted waves depending on the symmetry of final electronic state. For example, in the case of the $b^{3} \Sigma_{u}^{+}$state, the matrix element ( $k_{f}^{-} m|| \alpha k_{i}^{+}$) connects the $g$ and $u$ components of the two distorted waves, but still leaves the $\Sigma, \Pi, \Delta$, etc., symmetries uncoupled.

Equation (15) may be used to compute $T_{\mathrm{el}}\left(\overrightarrow{\mathrm{k}}_{f}-\overrightarrow{\mathrm{k}}_{\boldsymbol{i}}\right)$ for a given orientation of the molecule relative to the directions of $\vec{k}_{i}$ and $\vec{k}_{f}$. This is accomplished by solving Eq. (19) for the incident and final distorted waves, specifying the molecular orientation through the Fourier transforms. To compute the cross section we must perform the integral over molecular orientations in Eq. (14). We may write this integral as

$$
\int_{0}^{2 \pi} \int_{-1}^{1} d(\cos \theta) d \varphi\left|T_{\mathrm{el}}\right|^{2},
$$

the polar angles $\theta$ and $\varphi$ specifying the orientation of the molecular axis with respect to the axis perpendicular to the bisector of the angle between $\overrightarrow{\mathrm{k}}_{f}$ and $\vec{k}_{i}$ and lying in the plane of those vectors. The integration was performed in this coordinate system using six-point Gauss-Legendre quadrature in each of the variables $\cos \theta$ and $\varphi$. The integral is symmetric in $\varphi$ and need only be computed on the interval $[0, \pi / 2]$. At each orientation the planewave correction discussed in the previous section was made to $T_{\mathrm{el}}\left(\overrightarrow{\mathrm{k}}_{f}-\overrightarrow{\mathrm{k}}_{i}\right)$ and the corrected value was used in the integration over orientations.

Since we are ignoring the dependence of $T_{\mathrm{el}}$ $\left(\overrightarrow{\mathrm{k}}_{f}-\overrightarrow{\mathrm{k}}_{i}\right)$ on the internuclear separation, and thus on vibrational quantum numbers, the only factor left in the computation of the cross section is $\sum_{v^{\prime}}\left(k_{f} / k_{i}\right) q_{\nu \nu^{\prime}}$. Conservation of energy requires 


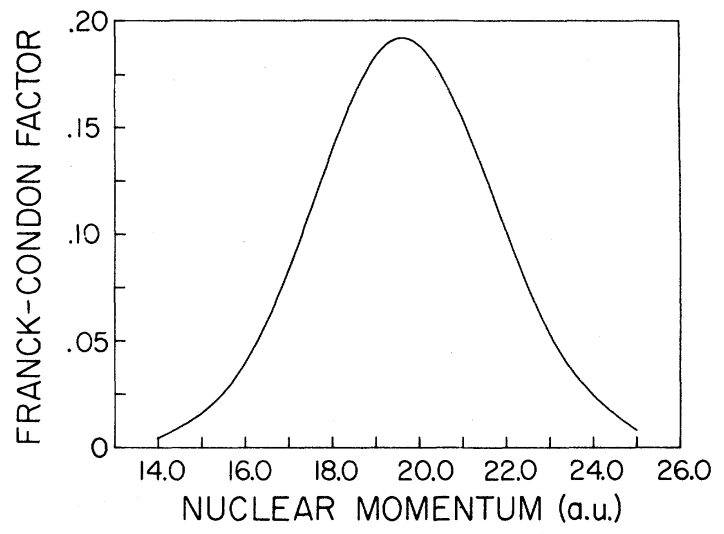

FIG. 1. Continuum Franck-Condon factor $q_{0 \nu}$, for the transition $X^{1} \Sigma_{g}^{+} \rightarrow b^{3} \Sigma_{u}^{+}$in molecular hydrogen. Normalization is chosen so that $\int_{0}^{\infty} q_{0 \nu}, d k_{\text {nuc }}=1$, where $k_{\text {nuc }}$ is the relative momentum of the dissociating protons in atomic units.

that $k_{f}$ depend on $\nu^{\prime} ; \nu$ was chosen as the lowest vibrational level of the ground state in all cases. The Franck-Condon factors for transitions to the discrete vibrational levels of the $a^{3} \Sigma_{g}^{+}$excited state were taken from the work of Cartwright and Kuppermann. ${ }^{24}$ No continuum vibrational states were included in the sum over $\nu^{\prime}$ for this state, because the sum of $q_{o \nu^{\prime}}$ for the first eight levels was sufficiently close to the expected value of unity.

The $b^{3} \Sigma_{u}^{+}$state is dissociative and thus has only continuum vibrational states. Vibrational wave functions were computed for this state by numerical integration using the potential-energy curve of

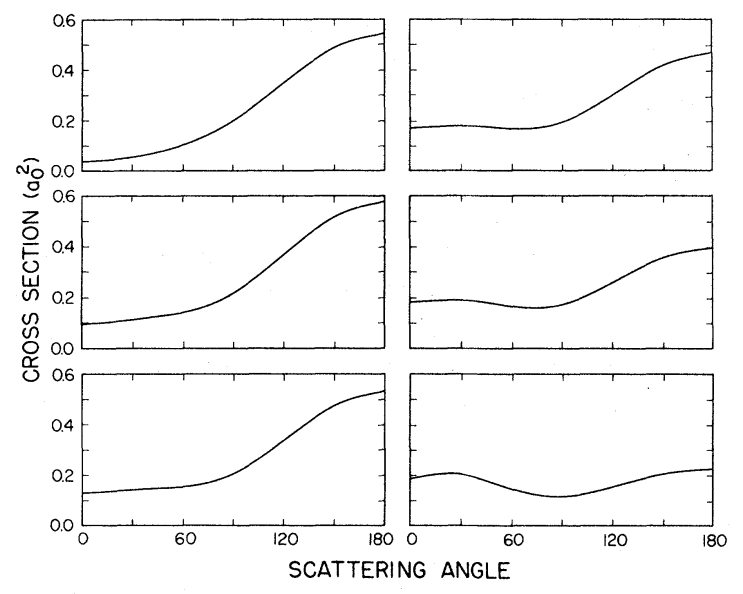

FIG. 2. Differential cross sections for the transition $X^{1} \Sigma_{g}^{+} \rightarrow b^{3} \Sigma_{u}^{+}$in molecular hydrogen. Cross sections are given in units of $a_{0}^{2}$. Curves on the left (running top to bottom) are for incident electron energies of 13 , 14, and $15 \mathrm{eV}$, respectively; curves on the right (top to bottom) are for electron energies of 16,17 , and 20 eV.
Kolos and Wolniewicz. ${ }^{12}$ The wave function of the lowest vibrational level of the ground state was also computed in this manner and the FranckCondon envelope is shown in Fig. 1. It was found to be in excellent agreement with the shape of the dissociative feature in the energy-loss spectrum for the $b^{3} \Sigma_{u}^{+}$state at $15-\mathrm{eV}$ incident energy in the unpublished experimental results of Srivastava and Trajmar. ${ }^{4}$ This agreement gives some indication that the neglect of the dependence of $T_{\mathrm{el}}\left(k_{f}-k_{i}\right)$ on the internuclear separation is justified in this case.

\section{RESULTS}

Differential cross sections at incident electron energies of $13,14,15,16,17$ and $20 \mathrm{eV}$ for excitation of the $b^{3} \Sigma_{u}^{+}$and $a^{3} \Sigma_{g}^{+}$states are shown in Figs. 2 and 3 , and Tables I and II, respectively. The DW-RPA gives similar angular distributions for both states. These cross sections are backward peaked at $13 \mathrm{eV}$ and develop a well-formed minimum at $90^{\circ}$ as the incident energy increases.

There is unfortunately little experimental data with which to compare these results. Srivastava and Trajmar ${ }^{4}$ have measured angular distributions for the excitation of the $b^{3} \Sigma_{u}^{+}$state at $15 \mathrm{eV}$ and their results agree very well with those predicted by the DW-RPA. The energy dependence of the differential cross sections we find for the $b^{3} \Sigma_{u}^{+}$state shows that the backward peaking becomes less pronounced as the energy increases and indicates the development of a maximum in the cross section near $30^{\circ}$ at higher energies. The experimental angular distribution of Trajmar et al.$^{25}$ for this transition does show a maximum between $30^{\circ}$ and $40^{\circ}$ for incident energies between 35 and $60 \mathrm{eV}$.

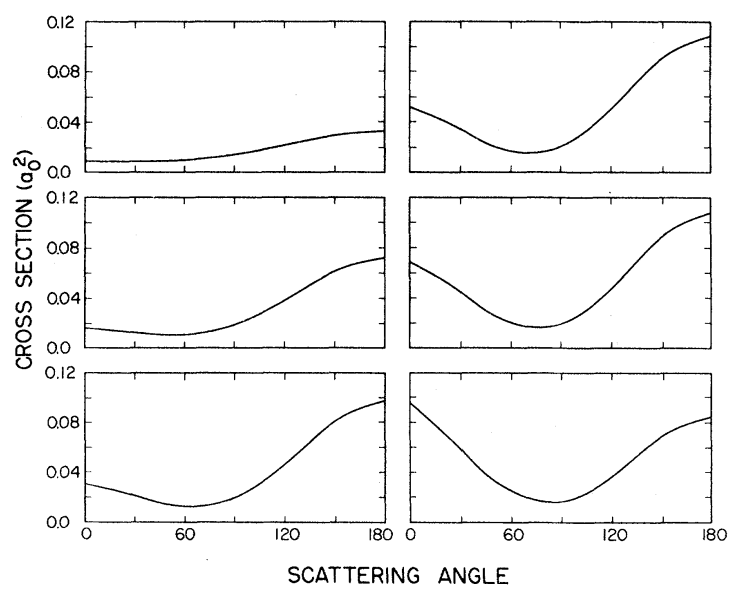

FIG. 3. Differential cross sections for the transition $X^{1} \Sigma^{1} \rightarrow a^{3} \Sigma_{g}^{+}$in molecular hydrogen. Cross sections are given in units of $a_{0}^{2}$. Incident electron energies are the same as in Fig. 2. 
TABLE I. Differential and total cross sections for the $X^{1} \Sigma_{g}^{+} \rightarrow b^{3} \Sigma_{u}^{+}$transition in molecular hydrogen. The differential cross sections are in units of $a_{0}^{2}$; total cross sections are in units of $\pi a_{0}^{2}$.

\begin{tabular}{|c|c|c|c|c|c|c|}
\hline $\begin{array}{c}\theta \\
(\mathrm{deg})\end{array} \backslash \begin{array}{c}E \\
(\mathrm{eV})\end{array}$ & 13 & 14 & 15 & 16 & 17 & 20 \\
\hline 0 & $3.682(-2)$ & $9.583(-2)$ & $1.284(-1)$ & $1.690(-1)$ & $1.819(-1)$ & $1.851(-1)$ \\
\hline 30 & $5.466(-2)$ & $1.128(-1)$ & $1.418(-1)$ & $1.777(-1)$ & $1.897(-1)$ & $2.037(-1)$ \\
\hline 45 & $7.453(-2)$ & $1.263(-1)$ & $1.478(-1)$ & $1.746(-1)$ & $1.815(-1)$ & $1.785(-1)$ \\
\hline 60 & $1.031(-1)$ & $1.409(-1)$ & $1.536(-1)$ & $1.669(-1)$ & $1.658(-1)$ & $1.464(-1)$ \\
\hline 90 & $2.009(-1)$ & $2.170(-1)$ & $2.044(-1)$ & $1.907(-1)$ & $1.718(-1)$ & $1.154(-1)$ \\
\hline 120 & $3.497(-1)$ & $3.685(-1)$ & $3.357(-1)$ & $2.962(-1)$ & $2.573(-1)$ & $1.531(-1)$ \\
\hline 135 & $4.253(-1)$ & $4.492(-1)$ & $4.103(-1)$ & $3.600(-1)$ & $3.101(-1)$ & $1.805(-1)$ \\
\hline 150 & $4.879(-1)$ & $5.167(-1)$ & $4.740(-1)$ & $4.150(-1)$ & $3.553(-1)$ & $2.041(-1)$ \\
\hline 180 & $5.455(-1)$ & $5.776(-1)$ & $5.327(-1)$ & $4.687(-1)$ & $3.964(-1)$ & $2.248(-1)$ \\
\hline $\begin{array}{c}\text { Total } \\
\text { cross section } \\
\left(\pi a_{0}^{2}\right)\end{array}$ & $9.317(-1)$ & 1.050 & 1.011 & $9.595(-1)$ & $8.757(-1)$ & $6.244(-1)$ \\
\hline
\end{tabular}

Cartwright ${ }^{25,26}$ has also calculated differential cross sections for the $b^{3} \Sigma_{u}^{+}$state using the OchkurRudge $^{27}$ (OR) approximation. This theory predicts a maximum near $40^{\circ}$ for incident energies between 15 and $85 \mathrm{eV}$. While this type of behavior does agree with the experimental measurements (which only extend to $80^{\circ}$ ) at energies above $35 \mathrm{eV},{ }^{25}$ it disagrees with the distributions we find below 20 $\mathrm{eV}$ in shape, although the magnitudes are similar. The Ochkur-Rudge approximation is, however, a high-energy theory based on first-order perturbation principles and should not be expected to be accurate at the low energies we have considered.

In the case of the $a^{3} \Sigma_{g}^{+}$excitation, no experimental data are available for the comparison. The energy-loss spectrum shows a strong overlap of the $a^{3} \Sigma_{g}^{+}$vibrational bands with those of the $c^{3} \Pi_{u}$ state and this interference makes angular distributions for the excitation of the $a^{3} \Sigma_{g}^{+}$state difficult to measure. $^{25}$ The only other results available for comparison are the OR calculations of Cartwright, ${ }^{25,26}$ and once again these differential cross sections do not agree with our results in shape, although the magnitudes are similar.

Since singlet-to-triplet radiative transitions are not observed in molecular hydrogen and the lowest triplet state is repulsive, electron impact excitation of any triplet state must lead eventually to dis sociation. Values for the dissociation cross section of $\mathrm{H}_{2}$, obtained by summing the total cross sections for excitation of the two lowest triplet states, are shown in Fig. 4. Also shown are the OR results of Cartwright, ${ }^{24,26}$ the Ochkur (O) calculations of Khare, ${ }^{28}$ and the experimental results

TABLE II. Differential and total cross sections for the $X^{1} \Sigma_{g}^{+} \rightarrow a^{3} \Sigma_{g}^{+}$transition in molecular hydrogen. The differential cross sections are in units of $a_{0}^{2}$; total cross sections are in units of $\pi a_{0}^{2}$.

\begin{tabular}{|c|c|c|c|c|c|c|}
\hline $\int \begin{array}{c}E \\
(\mathrm{eV})\end{array}$ & 13 & 14 & 15 & 16 & 17 & 20 \\
\hline 0 & $8.779(-3)$ & $1.599(-2)$ & $3.081(-2)$ & $5.170(-2)$ & $6.889(-2)$ & $9.632(-2)$ \\
\hline 30 & $8.611(-3)$ & $1.253(-2)$ & $2.145(-2)$ & $3.462(-2)$ & $4.534(-2)$ & $5.945(-2)$ \\
\hline 45 & $8.878(-3)$ & $1.073(-2)$ & $1.563(-2)$ & $2.368(-2)$ & $3.053(-2)$ & $3.939(-2)$ \\
\hline 60 & $9.784(-3)$ & $1.076(-2)$ & $1.277(-2)$ & $1.695(-2)$ & $2.071(-2)$ & $2.603(-2)$ \\
\hline 90 & $1.413(-2)$ & $1.876(-2)$ & $1.978(-2)$ & $2.012(-2)$ & $1.918(-2)$ & $1.664(-2)$ \\
\hline 120 & $2.153(-2)$ & $3.825(-2)$ & $4.661(-2)$ & $4.920(-2)$ & $4.687(-2)$ & $3.580(-2)$ \\
\hline 135 & $2.563(-2)$ & $5.033(-2)$ & $6.457(-2)$ & $6.985(-2)$ & $6.787(-2)$ & $5.251(-2)$ \\
\hline 150 & $2.926(-2)$ & $6.137(-2)$ & $8.128(-2)$ & $8.931(-2)$ & $8.789(-2)$ & $6.880(-2)$ \\
\hline 180 & $3.273(-2)$ & $7.217(-2)$ & $9.780(-2)$ & $1.086(-1)$ & $1.079(-1)$ & $8.522(-2)$ \\
\hline $\begin{array}{c}\text { Total } \\
\text { cross section } \\
\left(\pi a_{0}^{2}\right)\end{array}$ & $6.490(-2)$ & $1.067(-1)$ & $1.341(-1)$ & $1.530(-1)$ & $1.589(-1)$ & $1.486(-1)$ \\
\hline
\end{tabular}




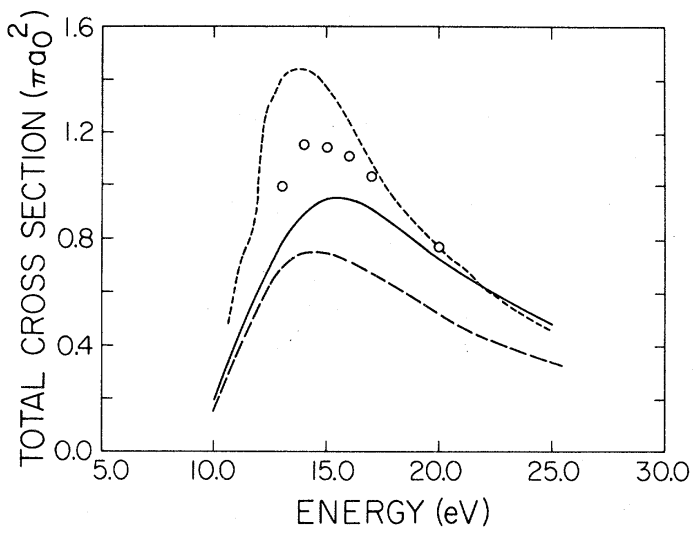

FIG. 4. Dissociation cross section of $\mathrm{H}_{2}$ into $2 \mathrm{H}$ by electron impact. Cross sections are in units of $\pi a_{0}^{2}$. $\bigcirc$, present results; solid line, experimental curve (Ref. 29), after subtraction of ionization cross section (see Refs. 30 and 24); short-dashed line, Ochkur-Rudge calculations (Ref. 24); long-dashed line, Ochkur calculation (Ref. 28). of Corrigan. ${ }^{29}$

At energies above $15.4 \mathrm{eV}$, Corrigan's data must be corrected to account for the effects of $\mathrm{H}_{2}$ ionization. The experimental curve shown in Fig. 4 thus represents the difference between Corrigan's measurement and the ionization cross section at the same impact energy measured by Harrison. ${ }^{24,30}$ Our results, as well as the theoretical results of Cartwright ${ }^{24,26}$ and Khare, ${ }^{28}$ show a peak in the ionization cross section between 14 and $15 \mathrm{eV}$, while the experimental data ${ }^{29}$ show this peak at $\sim 16 \mathrm{eV}$. Considering the possible errors involved in correcting Corrigan's data for the effects of ionization, the agreement between theory and experiment is satisfactory. There is also qualitative agreement between our total cross sections and the $O$ and $O R$ results. This agreement suggests that total cross sections computed using the $\mathrm{O}$ or OR approximation may be more reliable at low energies than the corresponding differential cross sections.
* Supported by a grant from the National Science Foundation.

$\dagger$ Present address: Lawrence Livermore Laboratory, University of California, Livermore, Calif. 94550.

\$Present address: Department of Chemistry, University of California, Berkeley, Calif. 94720 .

$\S_{\text {Contribution No. } 5167 .}$

"Work performed under the auspices of the U. S. Energy Research and Development Administration, Contract No. W-7405-ENG-48.

${ }^{1}$ M. R. H. Rudge, Advances in Atomic and Molecular Physics (Academic, New York, 1973), Vol. 9.

${ }^{2}$ D. G. Truhlar, D. C. Cartwright, and A. Kuppermann, Phys. Rev. 175, 113 (1968).

${ }^{3}$ L. D. Thomas, G. Csanak, H. S. Taylor, and B. S. Yarlagadda, J. Phys. B 7, 1719 (1974); for the $n=3$ levels of helium see A. Chutjian and L. D. Thomas, Phys. Rev. A 11, 1583 (1975).

${ }^{4}$ T. N. Rescigno, C. W. McCurdy, Jr., and V. McKoy, J. Phys. B (to be published).

${ }^{5}$ G. Csanak, H. S. Taylor, and R. Yaris, Phys. Rev. A 3 , 1322 (1971).

${ }^{6}$ T. $\mathrm{N}$. Rescigno, C. W. McCurdy, Jr., and V. McKoy, J. Phys. B $\underline{7}, 2396$ (1974).

${ }^{7}$ T. N. Rescigno, C. W. McCurdy, Jr., and V. McKoy, Chem. Phys. Lett. 27, 401 (1974).

${ }^{8}$ T. N. Rescigno, C. W. McCurdy, Jr., and V. McKoy, Phys. Rev. A 10, 2240 (1974).

${ }^{9}$ J. R. Taylor, Scattering Theory (Wiley, New York, 1972), p. 361.

${ }^{10}$ F. W. Read and G. L. Whiterod, Proc. Phys. Soc. Lond. 82, 434 (1963).

${ }^{11}$ A. Temkin and K. V. Vasavada, Phys. Rev. $\underline{160}, 109$ (1967).

${ }^{12}$ W. Kolos and L. Wolniewicz, J. Chem. Phys. $\underline{43}, 2429$
(1965).

${ }^{13}$ W. Kolos and L. Wolniewicz, J. Chem. Phys. $\underline{48}, 3672$ (1968).

${ }^{14}$ D. H. Madison and W. N. Shelton, Phys. Rev. A 7, 499 (1973).

${ }^{15}$ M. S. Pindzola and H. P. Kelly, Phys. Rev. A 11, 221 (1975).

${ }^{16}$ A. Messiah, Quantum Mechanics (North-Holland, Amsterdam, 1968), p. 839.

${ }^{17}$ T. Shibuya and V. McKoy, Phys. Rev. A 2 , 2208 (1970).

${ }^{18}$ See, for example, M. A. Crees and D. L. Moores, J. Phys. B 8 , L196 (1975).

${ }^{19}$ P. G. Burke and N. Chandra, J. Phys. B $\underline{5}, 1696$ (1972)

${ }^{20} \mathrm{P}$. G. Burke, N. Chandra, and F. A. Gianturco, Mol. Phys. 27, 1121 (1974).

${ }^{21}$ T. N. Rescigno, C. W. McCurdy, Jr., and V. McKoy, Phys. Rev. A 11, 825 (1975).

${ }^{22} \mathrm{~N}$. S. Ostlund (unpublished).

${ }^{23}$ S. Huzinaga, J. Chem. Phys. 42, 1293 (1965).

${ }^{24}$ D. C. Cartwright and A. Kuppermann, Phys. Rev. 163 , 86 (1967).

${ }^{25}$ S. Trajmar, D. C. Cartwright, J. K. Rice, R. T. Brinkmann, and A. Kuppermann, J. Chem. Phys. $\underline{49}$, 5464 (1968).

${ }^{26}$ D. C. Cartwright, Ph.D. thesis (California Institute of Technology, 1967) (unpublished).

${ }^{27}$ V. I. Ochkur, Zh. Eksp. Teor. Fiz. 45, 734 (1963) [Sov. Phys.-JETP 18, 503 (1964)]; M. R. H. Rudge, Proc. Phys. Soc. Lond. 85, 607 (1965); 86, 763 (1965). ${ }^{28}$ S. P. Khare, Phys. Rev. 157, 107 (1967).

${ }^{29}$ S. J. B. Corrigan, J. Chem. Phys. $\underline{43}, 4381$ (1965).

${ }^{30} \mathrm{H}$. Harrison, The Experimental Determination of Ionization Cross Sections of Gases Under Electron Impact (Catholic University of America, Washington, D. C., 1956), Table 4. 